\title{
Tratamiento del infarto agudo de miocardio en el Perú y su relación con eventos adversos intrahospitalarios: resultados del segundo registro peruano de infarto de miocardio con elevación del segmento ST (PERSTEMI-II)
}

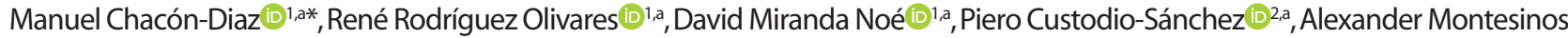

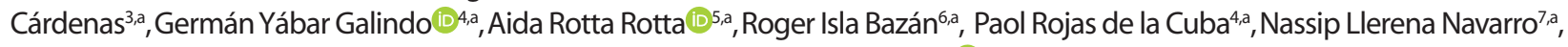

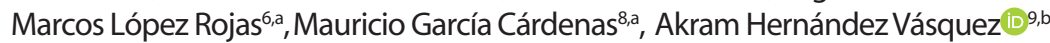

Recibido: 17 de abril 2021

Aceptado: 19 de mayo 2021

Filiación de los autores Instituto Nacional Cardiovascular INCOR, EsSalud. Lima, Perú. Hospital Nacional Almanzor Aguinaga Asenjo, EsSalud. Chiclayo, naga

Hospital Nacional Adolfo Guevara Velasco, EsSalud. Cusco, Perú. Velasco, EsSalud. Cusco, Perú.
Hospital Nacional Guillermo Hospital Nacional Guillermo
Almenara, EsSalud. Lima, Perú Hospital Nacional Cayetano Heredia, MINSA. Lima, Perú. Hospital Nacional Alberto Sabogal, EsSalud. Callao, Perú. Hospital Nacional Carlos Alberto Seguín Escobedo, EsSalud. Arequipa, Perú.

Hospital Hipólito Unanue, MINSA. Lima, Perú.

Universidad San Ignacio de Loyola. Lima, Perú.

Médico cardiólogo.

Médico investigador.

*Correspondencia

Calle coronel Zegarra 417, Jesús

María, Lima, Perú.

\section{RESUMEN}

Antecedentes. El infarto de miocardio con elevación del segmento ST (IMCEST), es una de las principales causas de morbimortalidad a nivel global, la reperfusión adecuada del miocardio consigue disminuir las complicaciones de esta entidad. El objetivo del estudio fue describir las características clínicas y terapéuticas del IMCEST en el Perú y la relación de la reperfusión exitosa con los eventos adversos intrahospitalarios. Materiales y métodos. Cohorte prospectiva, multicéntrica de pacientes con IMCEST atendidos durante el año 2020 en hospitales públicos del Perú. Se evaluaron las características clínicas, terapéuticas y eventos adversos intrahospitalarios, además de la relación entre la reperfusión exitosa del infarto y los eventos adversos. Resultados. Se incluyeron 374 pacientes, 69,5\% en Lima y Callao. La fibrinólisis fue usada en 37\% de casos (farmacoinvasiva $26 \%$ y sola $11 \%$ ), angioplastia primaria con $<12 \mathrm{~h}$ de evolución en $20 \%$, angioplastia tardía en $9 \%$ y $34 \%$ no accedieron a terapias de reperfusión adecuadas, principalmente por presentación tardía. El tiempo de isquemia fue mayor en pacientes con angioplastia primaria en comparación a fibrinólisis (mediana 7,7 h [RIQ 5-10] y $4 \mathrm{~h}$ [RIQ 2,3-5,5] respectivamente). La mortalidad fue de 8,5\%, la incidencia de insuficiencia cardiaca posinfarto fue de $27,8 \%$ y de choque cardiogénico de $11,5 \%$. El éxito de la reperfusión se asoció con menor mortalidad cardiovascular (RR: 0,28; IC95\%: 0,12-0,66, $\mathrm{p}=0,003$ ) y menor incidencia de insuficiencia cardiaca (RR: 0,61; IC95\%:0,43-0,85, p=0,004). Conclusiones. La fibrinólisis sigue siendo la terapia de reperfusión más frecuente en hospitales públicos del Perú. El menor tiempo de isquemia a reperfusión se asoció con el éxito de esta y, a su vez, a menores eventos adversos intrahospitalarios.
\end{abstract}

Correo

manuelchacon03@yahoo.es

Conflictos de interés

Ninguno.

Financiamiento

Autofinanciado.

Citar como:

Chacón-Diaz M, Rodríguez Olivares R, Miranda-Noé D, Custodio-Sánchez P, Montesinos Cárdenas A, Yabar Galindo G, et al. Tratamiento de infarto agudo de miocardio en el Perú y su relación con eventos adversos intrahospitalarios: resultados del Segundo Registro Peruano de Infarto de Miocardio con elevación del segmento ST (PERSTEMHII) Arch Peru Cardiol (P.
Palabras clave: Infarto del Miocardio; Fibrinólisis; Angioplastía; Mortalidad; Perú(fuente: DeCS BIREME).

\section{ABSTRACT}

Treatment of acute myocardial infarction in Peru and its relationship with in-hospital adverse events: results from the second peruvian registry of ST-segment elevation myocardial infarction (PERSTEMI-II).

\begin{abstract}
Background. ST-segment elevation myocardial infarction (STEMI), is an important cause of morbidity and mortality worldwide, and myocardial reperfusion, when adequate, reduces the complications of this entity. The aim of the study was to describe the clinical and treatment characteristics of STEMI in Peru and the relationship of successful reperfusion with in-hospital adverse events. Materials and methods. Prospective, multicenter cohort of STEMI patients attended during 2020 in public hospitals in Peru. We evaluated the clinical, therapeutic characteristics and in-hospital adverse events, also the relationship between successful reperfusion and adverse events. Results. A total of 374 patients were included, $69.5 \%$ in Lima and Callao. Fibrinolysis was used in $37 \%$ of cases (pharmacoinvasive $26 \%$ and fibrinolysis alone $11 \%$ ), primary angioplasty with $<12$ hours of evolution in $20 \%$, late angioplasty in $9 \%$ and $34 \%$ did not access adequate reperfusion therapies, mainly due to late presentation. Ischemia time was longer in patients with primary angioplasty compared to fibrinolysis (median 7.7 hours (RIQ 5-10) and 4 hours (RIQ 2.3-5.5) respectively). Mortality was 8.5\%, the incidence of postinfarction heart failure was $27.8 \%$ and of cardiogenic shock $11.5 \%$. Successful reperfusion was associated with lower cardiovascular mortality (RR:0.28; $95 \% \mathrm{Cl}: 0.12-0.66, \mathrm{p}=0.003$ ) and lower incidence of heart failure during hospitalization (RR: $0.61 ; 95 \% \mathrm{Cl}: 0.43-0.85, \mathrm{p}=0.004$ ). Conclusions. Fibrinolysis continues to be the most frequent reperfusion therapy in public hospitals in Peru. Shorter ischemia-to-reperfusion time was associated with reperfusion success, and in turn with fewer in-hospital adverse events.
\end{abstract}

Keywords: Myocardial Infarction; Fibrinolysis; Angioplasty; Mortality; Peru (source: MeSH NLM). 


\section{L} a Organización Mundial de la Salud (OMS) establece que la principal causa de muerte en el mundo es la enfermedad aterosclerótica y que alrededor del 30\% de las muertes reportadas se deben a cardiopatía isquémica ${ }^{(1)}$ cuyo impacto es mayor al de las enfermedades infecciosas y al cáncer, pronosticándose que para el 2030 la mortalidad se incrementará en $36 \%{ }^{(1,2)}$. Dentro de esta etiología, una de sus presentaciones más frecuentes es el infarto de miocardio con elevación del segmento ST (IMCEST). En los Estados Unidos, el IMCEST significa el $25-40 \%$ del total de casos de infarto de miocardio ${ }^{(3)}$, con una mortalidad intrahospitalaria del 5-6\% y del $7-18 \%$ al año del evento. Aproximadamente un 30\% son mujeres, $23 \%$ tienen diabetes mellitus y hasta un $7 \%$ no reciben terapia de reperfusión ${ }^{(4)}$.

Como parte del tratamiento, la reperfusión del miocardio en fase aguda modificó la historia natural del IMCEST debido a la reducción de la mortalidad y a la prevención o disminución de la ocurrencia de insuficiencia cardiaca secundaria a la necrosis miocárdica. La ventana temporal aceptada para la reperfusión del IMCEST es hasta las $12 \mathrm{~h}$ desde el inicio de síntomas; en situaciones clínicas especiales como inestabilidad hemodinámica o áreas de miocardio en riesgo muy extensas, se extiende más allá de $12 \mathrm{~h}^{(5)}$. Hay dos razones básicas por las que muchos pacientes no reciben reperfusión: la primera es por retraso y pérdida de la ventana de tiempo adecuada para obtener la reperfusión y la otra es la falta de un diagnóstico adecuado ${ }^{(6)}$.

En América Latina, según el registro ARGEN-IAMST (Argentina), el 83,5\% de pacientes con IMCEST reciben tratamiento de reperfusión (78,3\% mediante angioplastia primaria y $16 \%$ con fibrinolíticos) y tienen una mortalidad hospitalaria del $8,8 \%{ }^{(7)}$. Por su parte, el estudio RENASICA-II (México), identificó que el tratamiento de reperfusión fue en $32 \%$ por angioplastia coronaria y $37 \%$ por fibrinólisis, con una mortalidad hospitalaria del 10\% ${ }^{(8)}$. En el Perú, el año 2016, el registro PERSTEMI encontró que la fibrinólisis fue usada en $38 \%$ de casos, la angioplastia primaria en $29 \%$ y en $33 \%$ no recibieron reperfusión durante las primeras $12 \mathrm{~h}$ de evolución del IMCEST, en $12,9 \%$ se usó estrategia farmacoinvasiva, y la mortalidad intrahospitalaria fue de $10,1 \%{ }^{(9)}$.

Ante estos datos, el segundo registro nacional de infarto de miocardio PERSTEMI-Il, buscó evaluar la evolución del perfil epidemiológico del IMCEST en el Perú cuatro años después del primer registro, conocer las estrategias de reperfusión más prevalentes en nuestro medio, dar a conocer las principales complicaciones del IMCEST y los eventos adversos en el seguimiento al año. En este artículo se describen las características de presentación y tratamiento del IMCEST, y la relación de la reperfusión exitosa con los eventos adversos intrahospitalarios.

\section{Materiales y métodos}

El registro PERSTEMI-II es una cohorte prospectiva y multicéntrica de pacientes con IMCEST atendidos en hospitales públicos de mayor nivel (del Ministerio de Salud y EsSalud) en las capitales de departamento del Perú. El protocolo de estudio incluyó a todos los pacientes mayores de 18 años con diagnóstico de IMCEST según la cuarta definición universal de infarto de miocardio atendidos durante el año 2020, en quienes se evaluaron las características clínicas, diagnósticas y de tratamiento del infarto, así como las complicaciones y la mortalidad intrahospitalaria. Se excluyeron pacientes con infarto de miocardio sin elevación del segmento ST (IMSEST), IMCEST no persistente y pacientes con síndrome de Takotsubo.

La recolección de datos (previo consentimiento informado) se hizo directamente de la historia clínica a una base electrónica diseñada para dicho fin (http://40.77.71.10/ www/Perstemi2/). Las variables de estudio incluyeron: variables generales (edad, sexo, lugar de atención); epidemiológicas (antecedentes patológicos, factores de riesgo cardiovascular); clínicas (síntomas, características del electrocardiograma, clasificación Killip Kimbal); acceso a reperfusión, tipo de reperfusión, tiempos de primer contacto médico y tiempo de isquemia a reperfusión, tratamiento intrahospitalario y al alta, complicaciones intrahospitalarias y mortalidad (intrahospitalaria y a los 30 días del evento).

Se consideró tratamiento de reperfusión a la administración de alguna terapia de este tipo en las primeras 12 h del infarto; paciente con reperfusión tardía si la intervención coronaria percutánea primaria (ICPp) se realizó entre las 12 a 48 h, y paciente sin acceso a reperfusión a aquel que no recibió tratamiento alguno de reperfusión ${ }^{(6)}$. Se consideró éxito de reperfusión con fibrinolisis a la caída del segmento ST > 50\% a los 90 min de iniciado el fármaco, y con ICPp al flujo TIMI 3 de la arteria responsable del infarto (ARI) posintervención ${ }^{(3,6)}$ en ambos casos se consideró como caso «reperfundido» para el análisis estadístico, de lo contrario, o en caso del paciente sin acceso a reperfusión, se consideró como «no reperfundido».

Las variables categóricas fueron expresadas en frecuencias y porcentajes, las variables numéricas en promedios o medianas y sus respectivas medidas de dispersión según su distribución. La evaluación de la asociación entre dos variables categóricas se realizó mediante la prueba de chi cuadrado, y entre variables numéricas con la prueba de t de Student (distribución normal) o U de Mann-Whitney (distribución no paramétrica). Se realizaron modelos lineales generalizados de la familia binomial con función de enlace log para estimar el riesgo relativo (RR) crudo y ajustado y sus respectivos IC al $95 \%$ de los factores asociados 
a reperfusión exitosa y del impacto de esta en la frecuencia de eventos adversos intrahospitalarios. La evaluación estadística se realizó usando el programa Stata 14.0 (StataCorp, College Station, Texas, USA).

\section{Resultados}

Se invitó a 25 centros hospitalarios de mayor nivel resolutivo del sector público a nivel nacional a participar en el registro, de ellos 17 participaron activamente en la recolección de casos. Desde el 1 de enero al 31 de diciembre de 2020; se registraron en el sistema un total de 405 casos de los cuales se excluyeron a los casos incompletos o con diagnósticos erróneos, quedando 374 pacientes que conformaron la población de estudio.

El 69,5\% fueron registrados en Lima y Callao, 88,5\% fueron atendidos en centros del seguro social de EsSalud y 11,5\% en centros del Ministerio de Salud (MINSA). El 67,4\% de casos fueron referidos a centros de mayor complejidad para completar su tratamiento de reperfusión. El número de casos fue disminuyendo durante el año por la influencia de la pandemia por SARS-CoV-2 (Figura 1). El $85 \%$ de casos fueron varones y la mediana de edad fue de 66 años (RIQ: 58-74 años); la edad de presentación en mujeres fue mayor a la de los varones (71 y 65 años, respectivamente, $p=0,01$ ). Los antecedentes de riesgo más frecuentes en la población fueron hipertensión arterial y dislipidemia (Tabla 1).
Tabla 1. Antecedentes y factores de riesgo de la población en estudio

\begin{tabular}{lcc}
\hline Antecedentes & $\mathbf{n}$ & $\%$ \\
\hline Hipertensión arterial & 198 & 52,9 \\
Dislipidemia & 198 & 52,9 \\
\hline Diabetes mellitus tipo 2 & 111 & 29,7 \\
\hline Tabaquismo & 81 & 21,6 \\
\hline Infarto de miocardio & 28 & 7,5 \\
\hline Enfermedad renal crónica & 28 & 7,5 \\
\hline Síndrome coronario crónico & 24 & 6,4 \\
Evento cerebro vascular & 18 & 4,8 \\
\hline Hiperuricemia & 12 & 3,2 \\
\hline Revascularización miocárdica & 12 & 3,2 \\
\hline Falla cardiaca & 9 & 2,4 \\
\hline
\end{tabular}

\section{Presentación clínica}

La manifestación inicial fue en 93,8\% angina típica; 29\% disnea; 5,3\% dolor torácico atípico: 4\% síncope y 3,2\% arresto cardiaco. Las mujeres tuvieron mayor proporción de síntomas atípicos que los varones (disnea 36,4 y $27,9 \%$; síncope 5,5 y 3,8\% y dolor torácico atípico 9,1 y 4,7\% respectivamente) aunque sin significancia estadística.

El primer electrocardiograma encontró un $92,5 \%$ de casos en ritmo sinusal; $4,5 \%$ con bloqueo aurículoventricular

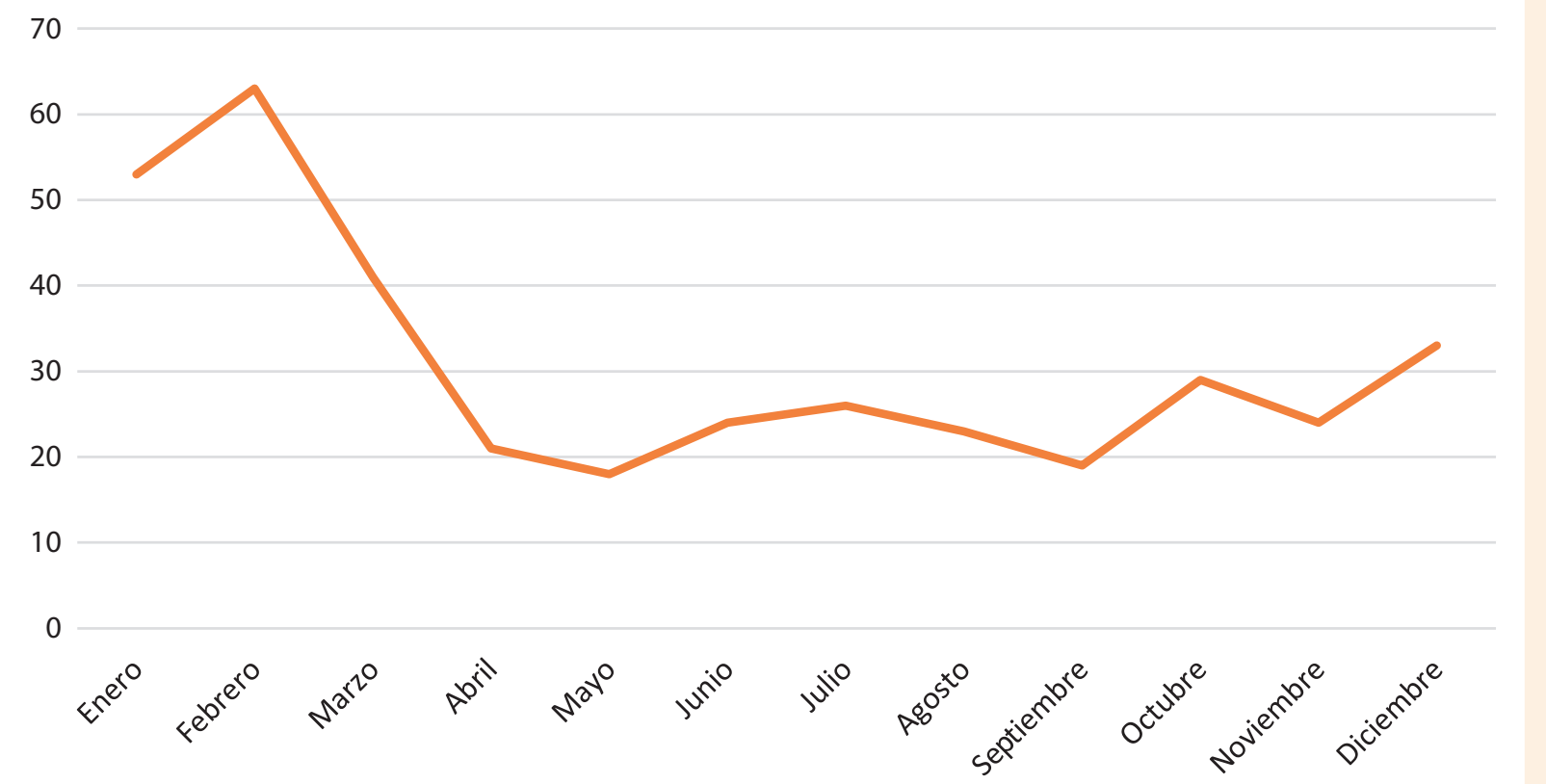

Figura 1. Número de casos de IMCEST reportados por mes durante el año 2020 
de alto grado y 2,9\% con fibrilación auricular. La localización más frecuente del infarto fue en la pared anterior (anteroseptal, anterolateral y anterior sola) en un $38,2 \%$, seguida por la cara inferior (26,5\%), anterior extenso (18,7\%), ínferolateral (14,2\%) y lateral (2,4\%). El estado clínico al ingreso en el $65,5 \%$ de casos fue en Killip Kimbal (KK) I; 27,3\% en KK II; 2,9\% en KK III y 4,3 en KK IV. Con el paso de las horas el 59,4\% permaneció en KK I; $27 \%$ en KK II; $4 \%$ empeoró a estadio KK III y 9,6\% a KK IV. En general, el tiempo a primer contacto médico fue de 2,5 h (RIQ: 1 - 6) y el tiempo de isquemia a reperfusión de 5,3 h (RIQ: 3 - 9).

\section{Estrategias de reperfusión (Figura central)}

Doscientos cinco pacientes $(55 \%$ de la población de estudio) recibieron algún tipo de reperfusión en las primeras 12 h de evolución del infarto, 131 (64\%) fibrinólisis y $74(36 \%)$ ICPp.

Treinta y un pacientes $(8,2 \%)$ recibieron reperfusión tardía pasadas las $12 \mathrm{~h}$ hasta las $24 \mathrm{~h}$ del infarto (3 fibrinólisis y 28 ICPp). Los motivos fueron: falla cardiaca (15 casos), infartos grandes (anterior extenso o inferolateral) sin falla cardiaca (8 casos) y desconocido (8 casos). En 6 pacientes (1,6\%) la ICPp se realizó con más de 24 h de evolución hasta 48 horas (en 5 por falla cardiaca posinfarto). En cuatro casos no se consignó el tiempo de isquemia a la reperfusión (3 fibrinolisados y 1 PCIp). Ciento veintiocho pacientes (34\%) no accedieron a ninguna terapia de reperfusión, principalmente por presentación tardía (Figura 2).

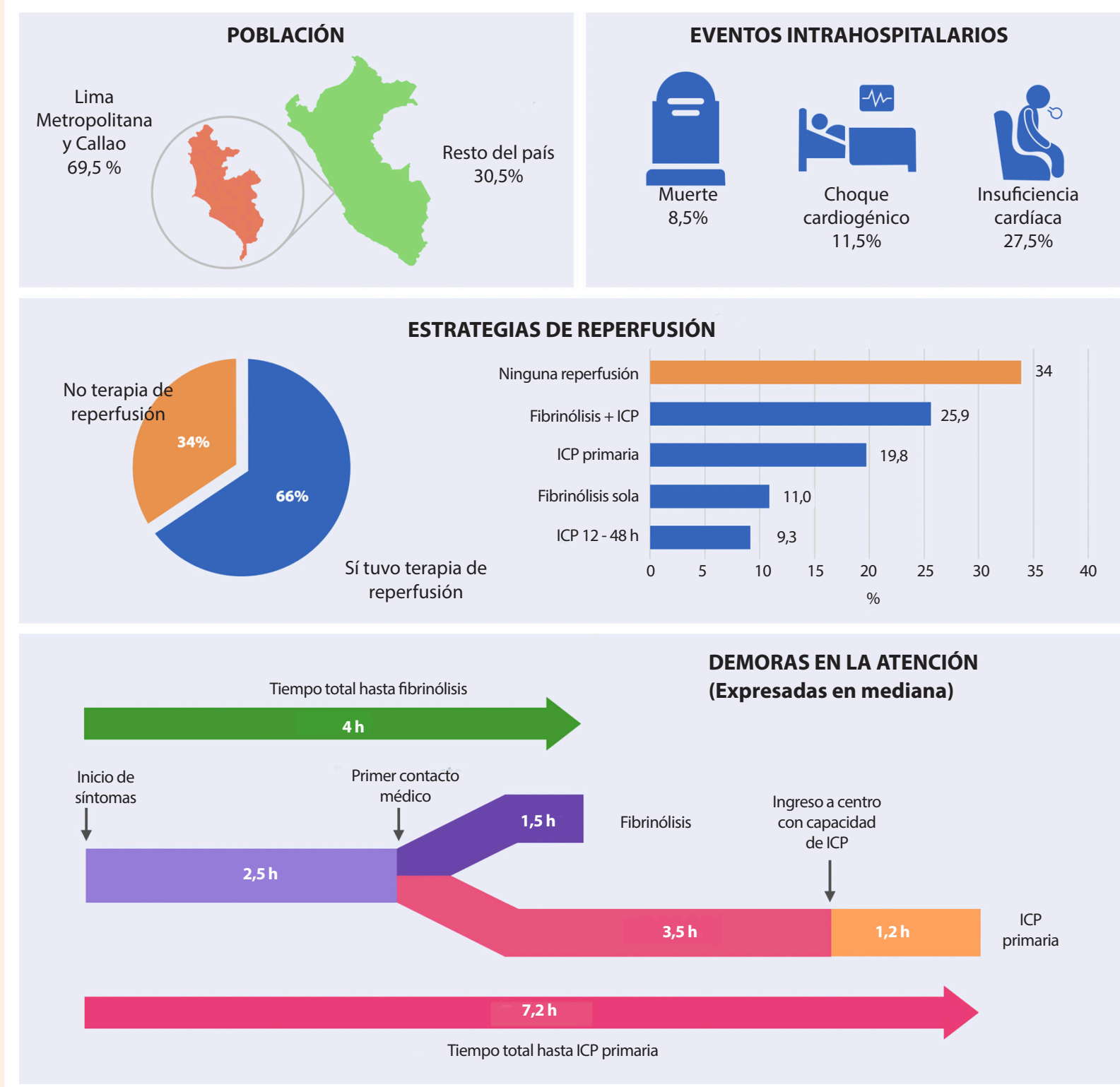

Figura central. Reperfusión, demoras en la atención y eventos intrahospitalarios en IMCEST - Registro PERSTEMI II. Perú 2020. 
35

30,9

30

25

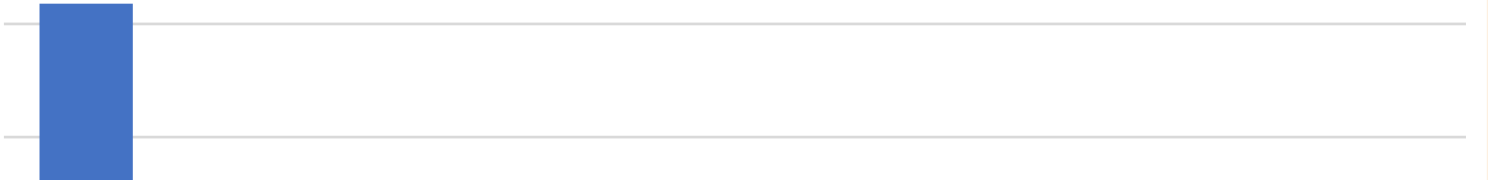

20

20,3

15

10

5

0

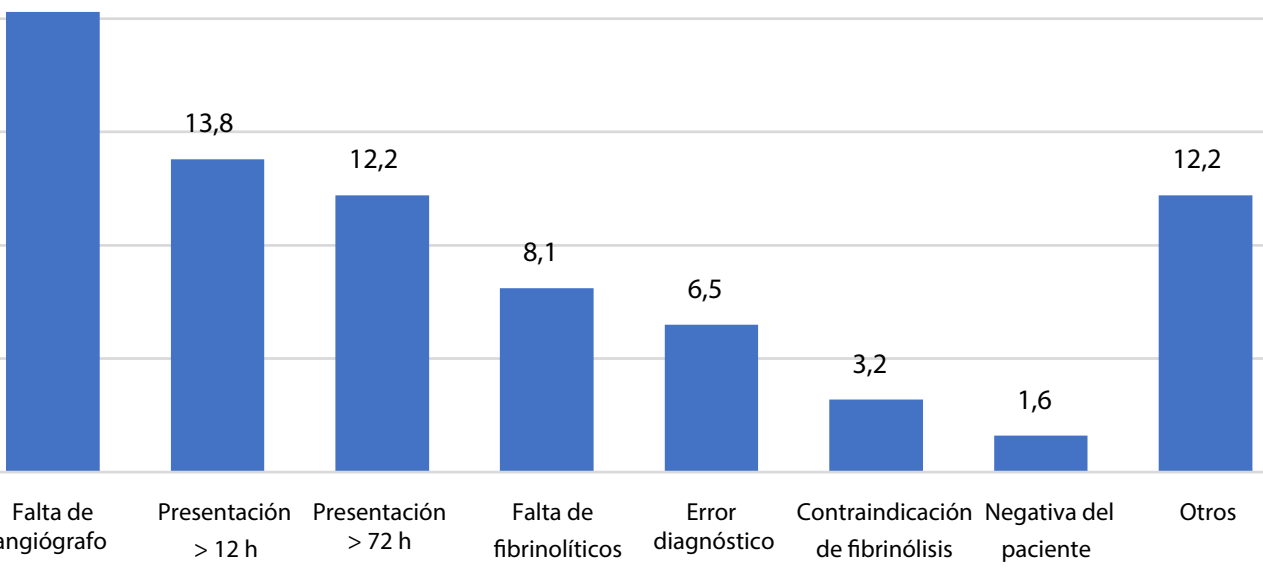

Figura 2. Motivos de no aplicación de terapias de reperfusión en IMCEST (valores expresados en porcentajes)

\section{Fibrinólisis}

Ciento treinta y siete pacientes (37\% de la población) fueron fibrinolisados como primera estrategia de reperfusión. En todos los casos con alteplase, y representa el tratamiento de reperfusión más frecuente (56\%). La tasa de éxito fue de 66\% (90 pacientes).

Como parte de una estrategia farmacoinvasiva luego de una fibrinólisis exitosa, el 77\% (69 pacientes) fueron a coronariografía más intervención coronaria percutánea (ICP) entre las 3 a $24 \mathrm{~h}$ (estrategia de ICP temprana de rutina), lo que significa un $50 \%$ de todos los fibrinolisados y $18 \%$ del total de pacientes del estudio. El éxito de la ICP en este grupo fue de $99 \%$. En los 47 casos donde la fibrinólisis no fue exitosa, se realizó ICP de rescate en $28(60 \%)$, lo que representa el $7,5 \%$ del total de casos del estudio.

De lo anterior se desprende que $29 \%$ de pacientes fibrinolisados (40 casos) no accedieron a una terapia invasiva posterior (la mayoría en el interior del país y en hospitales del MINSA) que representa el $11 \%$ de la población de estudio.

\section{Intervención coronaria percutánea primaria (ICPp)}

La ICPp en las primeras $12 \mathrm{~h}$ de evolución se realizó en 74 pacientes (19,8\% de la población) y su tasa de éxito angiográfico fue del 73\%. En 34 pacientes (9\% de la población) se realizó ICPp tardía, la tasa de éxito en este caso fue del $54 \%(p=0,078)$. Los tiempos de primer contacto médico, tiempo de isquemia a ICPpy tiempo puerta balón se detallan en la (Tabla 2).

En las coronariografías se encontró que la ARI en el $62 \%$ fue la descendente anterior, 31\% la coronaria derecha, 6\% la circunfleja y en $1 \%$ no se identificó ARI (MINOCA). Los porcentajes de flujo TIMI 0, 1, 2 y 3 pre-ICP fueron 33\%, 18\%, 21\% y 28\%, respectivamente; pos-ICP fue de $4 \%, 7 \%, 13 \%$ y $76 \%$. Se colocaron stents en el $92 \%$ de casos que ingresaron a coronariografía, en su mayoría stents liberadores de fármacos (96\%). El 51\% de pacientes (104 casos) tenían enfermedad coronaria multiarterial, de ellos, en el $65 \%$ se realizó intervención en otras arterias diferentes a

Tabla 2. Demoras en el tratamiento de reperfusión según estrategia usada en las primeras $12 \mathrm{~h}$ de evolución

\begin{tabular}{lcccc}
\hline & \multicolumn{2}{c}{ Fibrinólisis } & \multicolumn{2}{c}{ ICP primaria } \\
\hline & $\begin{array}{c}\text { Mediana } \\
\text { (horas) }\end{array}$ & RIQ & $\begin{array}{c}\text { Mediana } \\
\text { (horas) }\end{array}$ & RIQ \\
\hline TPCM & 1,5 & $0,7-3$ & 2 & $1-4$ \\
TTI & 4 & $2,3-5,5$ & 7,7 & $5-10$ \\
TPA/TPB & 1,5 & $0,7-2,6$ & 1,2 & $1-1,5$ \\
\hline
\end{tabular}

ICP: intervención coronaria percutánea. TPCM: primer contacto médico. TTI: tiempo total de isquemia a reperfusión. TPA: tiempo puerta-aguja. TPB: tiempo puerta-balón. RIQ: rango intercuartil. 
la ARI; $26 \%$ en el mismo procedimiento y $73 \%$ diferida antes del alta (97\% de rutina y solo $3 \%$ guiada por estudios de isquemiaviabilidad). Solo $17(4,5 \%)$ fueron sometidos a cirugía cardiaca; de este grupo, 6 por ICP frustra, 5 por choque cardiogénico, 4 por anatomía de alto riesgo para ICP y 2 por complicación mecánica.

Un 26\% de pacientes que accedieron a alguna terapia no tuvieron éxito en la reperfusión; al sumarlos con los pacientes sin acceso a reperfusión, encontramos que 192 (51,3\% de la población) no tuvieron reperfusión adecuada del miocardio. Al realizar el análisis de los factores asociados con una reperfusión exitosa, encontramos en el modelo ajustado que dos factores estaban asociados con esta: la atención en un centro hospitalario de EsSalud (RR: 2,12, p=0,006, IC95\%: 1,23-3,65) y el tiempo total de isquemia hasta reperfusión < 6 h (RR: 1,60, IC95\%:1,12 - 2,28, $\mathrm{p}=0,010$ comparado con un tiempo $>12 \mathrm{~h}$ ) (Tabla 3).

\section{Medicación y eventos adversos intrahospitalarios}

La estancia hospitalaria en promedio fue de 7 días (RIQ:511). El uso de doble antiagregación plaquetaria llegó al 95\%, betabloqueadores $81 \%$, IECA/ARA2 69\%, estatinas 94\%, diuréticos $21 \%$ y antialdosterónicos $25 \%$.

La mortalidad intrahospitalaria fue de 8,6\% (32 casos), $6,7 \%$ de causa cardiaca y $1,8 \%$ de causa no cardiaca. Se encontró una incidencia de falla cardiaca posinfarto de $27,8 \%$; de choque cardiogénico de 11,5\%; angina posinfarto 7,8\%; complicación mecánica del infarto 2,9\%; arresto cardiaco 8,5\%; accidente cerebro vascular $0,8 \%$ y sangrado mayor $2,9 \%$. Es importante mencionar que 16 pacientes $(4,2 \%)$ fueron atendidos en contexto de infección activa por SARS-COv2. La mortalidad en el seguimiento a los 30 días fue de 9,1\% (34 casos).

El éxito de la reperfusión fue la variable que influyó notablemente en la existencia de eventos adversos intrahospitalarios, tanto en el análisis crudo como en el ajustado a edad, sexo y enfermedades de base (Tabla 4).

\section{Discusión}

La información obtenida de los registros clínicos y su comparación temporal se han convertido en herramientas fundamentales con el objetivo de mejorar la calidad de la atención de los pacientes con IMCEST ${ }^{(10)}$. El precepto de que no se puede mejorar lo que no se puede medir describe el rol central que los registros han tenido en la reducción de la mortalidad de los pacientes con IMCEST a nivel mundial ${ }^{(11)}$. El registro PERSTEMI II nos permite conocer y comparar las características epidemiológicas y resultados clínicos de los pacientes con IMCEST respecto a lo reportado por PERSTEMI I (2016-2017) ${ }^{(9)}$.
Encontramos una reducción en el número de pacientes atendidos durante el año de estudio, lo que se produjo en el contexto de la pandemia por COVID-19 que ha generado la reducción de los casos de IMCEST en todo el mundo, especialmente al inicio de la pandemia (disminución del 59\% en el número de ingresos al inicio del estado de emergencia en el Perú) y en probable relación al temor de los pacientes al contagio al acudir a los centros hospitalarios ${ }^{(12-14)}$. La mayoría de casos fueron reportados en las ciudades de Lima y el Callao y en centros del Seguro Social de EsSalud, lo que se repite respecto a lo observado en PERSTEMI I.

Con relación a los factores de riesgo cardiovascular, los más frecuentes fueron la hipertensión arterial y la dislipidemia, observándose un marcado incremento en la frecuencia de esta última respecto a lo reportado por PERSTEMI I (52,9\% vs 34,9\%) lo que también se reporta en otros registros latinoamericanos (15-17).

La angina típica y la disnea siguen siendo las formas más frecuentes de presentación y los síntomas atípicos más prevalentes en mujeres. Con relación a la localización del infarto, sigue siendo la cara anterior la más prevalente a semejanza de lo reportado por los registros mexicanos RENASICA III y PHASE$M X$, pero distinto a lo evidenciado por los registros ARGEN-IAMST y RESISST, argentino y brasileño respectivamente, donde el compromiso de la cara inferior fue el más frecuente $(15,16,18,19)$.

Destacamos la progresión del deterioro hemodinámico en cerca del $10 \%$ de los pacientes que desarrollan choque cardiogénico en hospitalización luego del ingreso hospitalario, cifra mayor a la reportada por Farré et al. durante el registro Codi IAM ${ }^{(20)}$. Dada la alta mortalidad asociada con esta condición, especialmente en nuestro país como lo reporta un reciente registro unicéntrico nacional; es importante la identificación, prevención y seguimiento de estos pacientes ${ }^{(21,22)}$.

Con relación a PERSTEMI I, hubo una reducción en el número de pacientes sometidos a algún tipo de terapia de reperfusión en las primeras 12 h (55\% vs 67\%) siendo la presentación tardía el principal motivo de forma recurrente. Esta tasa de reperfusión se encuentra por debajo de la reportada por registros europeos como FAST AMI con $77 \%$ o sudamericanos como ARGEN-IAM-ST con 88\%, pero por encima de la evidenciada por otros registros latinoamericanos como RESISST con $40,7 \%$ y RENASICA III con $52,6 \%{ }^{(15,16,18,23)}$. Estas diferencias expresan las distintas realidades organizativas de los sistemas de salud y aspectos logísticos para ofrecer terapia de reperfusión. En el Perú existe una única sala de hemodinámica pública con atención permanente para el manejo de pacientes con IMCEST, en el Instituto Nacional Cardiovascular, realidad que no se ha modificado desde lo reportado hace 4 años.

En PERSTEMI I el tiempo a primer contacto médico fue de 2 h (RIQ:1- 4,5) mientras que en PERSTEMI II fue de 2,5 h (RIQ 1-6), 
Tabla 3. Factores asociados al éxito de la terapia de reperfusión del IMCEST

\begin{tabular}{|c|c|c|c|c|}
\hline \multirow[t]{2}{*}{ Característica } & \multicolumn{2}{|l|}{ Modelo crudo } & \multicolumn{2}{|l|}{$\begin{array}{l}\text { Modelo } \\
\text { ajustado* }\end{array}$} \\
\hline & RR (IC 95\%) & Valor $\mathbf{p}$ & RRa (IC 95\%) & Valor $p$ \\
\hline \multicolumn{5}{|l|}{ Sexo } \\
\hline Femenino & Referencia & & & \\
\hline Masculino & $0,76(0,53-1,08)$ & 0,122 & $0,95(0,74-1,24)$ & 0,718 \\
\hline Edad (años) & $1,00(0,99-1,00)$ & 0,405 & No incluida & \\
\hline \multicolumn{5}{|l|}{ Tabaquismo } \\
\hline No & Referencia & & & \\
\hline Sí & $1,33(1,07-1,65)$ & 0,009 & $1,07(0,92-1,23)$ & 0,381 \\
\hline \multicolumn{5}{|l|}{ Diabetes mellitus } \\
\hline No & Referencia & & & \\
\hline Sí & $0,82(0,64-1,06)$ & 0,127 & $1,02(0,87-1,21)$ & 0,728 \\
\hline \multicolumn{5}{|l|}{ Hipertensión arterial } \\
\hline No & Referencia & & Referencia & \\
\hline Sí & $0,83(0,68-1,02)$ & 0,084 & $0,97(0,83-1,12)$ & 0,640 \\
\hline \multicolumn{5}{|l|}{ Enfermedad renal crónica } \\
\hline No & Referencia & & No incluida & \\
\hline Sí & $0,87(0,56-1,36)$ & 0,544 & & \\
\hline \multicolumn{5}{|l|}{ Falla cardiaca crónica } \\
\hline No & Referencia & & No incluida & \\
\hline Sí & $0,68(0,27-1,72)$ & 0,416 & & \\
\hline \multicolumn{5}{|c|}{ Lugar de establecimiento de salud } \\
\hline MINSA & Referencia & & Referencia & \\
\hline EsSalud & $2,50(1,38-4,51)$ & 0,002 & $2,12(1,23-3,65)$ & 0,006 \\
\hline \multicolumn{5}{|l|}{ Lugar de atención } \\
\hline Fuera de Lima & Referencia & & Referencia & \\
\hline Lima & $1,26(0,98-1,62)$ & 0,069 & $1,04(0,86-1,27)$ & 0,676 \\
\hline \multicolumn{5}{|l|}{ Traslado } \\
\hline No & Referencia & & Referencia & \\
\hline Sí & $1,35(1,05-1,73)$ & 0,018 & $0,93(0,76-1,14)$ & 0,505 \\
\hline \multicolumn{5}{|l|}{ Tiempo de isquemia } \\
\hline$<6$ horas & $1,46(1,11-1,92)$ & 0,007 & $1,60(1,12-2,28)$ & 0,010 \\
\hline $6-12$ & $1,29(0,96-1,75)$ & 0,096 & $1,34(0,95-1,89)$ & 0,099 \\
\hline$>12$ horas & Referencia & & Referencia & \\
\hline Tiempo de primer contacto & $0,91(0,88-0,94)$ & $<0,001$ & $1,01(0,98-1,04)$ & 0,525 \\
\hline
\end{tabular}

hallazgo que podría interpretarse como expresión de la ausencia de programas de educación a nivel nacional para el reconocimiento temprano de los síntomas del infarto y la búsqueda de atención médica, así como debido a la pandemia por COVID-19 que motivó la búsqueda tardía de atención hospitalaria por el temor al contagio ${ }^{(24,25)}$. La demora en la aplicación de la fibrinólisis $(1,5$ h) y de la ICPp (4,7 h) luego del primer contacto médico (demora del sistema), evidencia la inoperancia del sistema de salud público en el tratamiento del IMCEST.

Similar a lo reportado en PERSTEMI I, la fibrinólisis en las primeras $12 \mathrm{~h}$ continuó siendo la estrategia de reperfusión más 
Tabla 4. Asociación entre la reperfusión exitosa del IMCEST y desenlaces intrahospitalarios

\begin{tabular}{|c|c|c|c|c|c|c|}
\hline \multirow[t]{2}{*}{ Característica } & \multirow{2}{*}{$\begin{array}{c}\text { Reperfundido } \\
n=182(48,7)\end{array}$} & \multirow{2}{*}{$\begin{array}{c}\text { No } \\
\text { reperfundido }\end{array}$} & \multirow{2}{*}{$\begin{array}{l}\text { Modelo crudo } \\
\text { RR (IC 95\%) }\end{array}$} & \multicolumn{3}{|c|}{$\begin{array}{c}\text { Modelo } \\
\text { ajustado* }\end{array}$} \\
\hline & & & & Valor p & RRa (IC 95\%) & Valor p \\
\hline Mortalidad general & $6(3,3)$ & $26(13,5)$ & $0,24(0,10-0,58)$ & 0,001 & $0,28(0,12-0,66)$ & 0,003 \\
\hline Mortalidad cardiovascular & $5(2,8)$ & $20(10,4)$ & $0,26(0,10-0,69)$ & 0,007 & $0,31(0,13-0,74)$ & 0,009 \\
\hline Mortalidad no cardiaca & $1(0,6)$ & $6(3,1)$ & $0,18(0,02-1,45)$ & 0,106 & $0,17(0,02-1,55)$ & 0,116 \\
\hline Falla cardiaca sintomática & $36(19,8)$ & $68(35,4)$ & $0,56(0,39-0,79)$ & 0,001 & $0,61(0,43-0,85)$ & 0,004 \\
\hline Choque cardiogénico & $10(5,5)$ & $33(17,2)$ & $0,32(0,16-0,63)$ & 0,001 & $0,35(0,18-0,68)$ & 0,002 \\
\hline Angina posinfarto & $7(3,9)$ & $22(11,5)$ & $0,34(0,15-0,77)$ & 0,010 & $0,33(0,14-0,80)$ & 0,014 \\
\hline Compliación mecánica & $2(1,1)$ & $9(4,7)$ & $0,23(0,05-1,07)$ & 0,062 & $0,36(0,08-1,65)$ & 0,188 \\
\hline Arresto cardiaco & $9(5,0)$ & $23(12,0)$ & $0,41(0,20-0,87)$ & 0,020 & $0,47(0,23-0,96)$ & 0,038 \\
\hline Sangrado mayor ${ }^{* *}$ & $4(2,2)$ & $7(3,7)$ & $0,60(0,18-2,03)$ & 0,414 & $0,85(0,25-2,91)$ & 0,802 \\
\hline
\end{tabular}

utilizada (37\% vs 38\%). Sin embargo, encontramos una disminución en el porcentaje de ICPp (19,8\% vs $29 \%$ ) lo cual puede explicarse por la influencia de la pandemia por COVID-19 sobre la decisión del tipo de reperfusión a emplear, pues la estrategia de fibrinólisis implica menor demanda logística y ha sido recomendada por algunas instituciones científicas ${ }^{(26)}$. Esto va de la mano con lo reportado por De Luca et al. ${ }^{(27)}$ que encontraron una reducción de $19 \%$ en las ICPp durante la pandemia por COVID-19 en 40 centros europeos de alto volumen de procedimientos.

Además de la reducción de la ICPp como terapia de reperfusión, el porcentaje de pacientes con resultado angiográfico flujo TIMI III disminuyó marcadamente en relación a PERSTEMI I (67\% vs $82 \%$ ) lo que puede ser explicado por la intervención de un mayor número de pacientes de presentación tardía ( $>12 \mathrm{~h}$ del inicio de los síntomas) en el presente registro.

La tasa de éxito de la fibrinólisis se mantuvo cercana al $70 \%$, lo que reafirma la utilidad de dicha estrategia en el contexto actual. A pesar de la sobrecarga de los sistemas de salud a nivel nacional y mundial, no se incrementó el tiempo total de isquemia a reperfusión (5,3 h vs 6 h en PERSTEMI I) lo que podría explicarse por el aumento en el uso de la fibrinólisis por encima de la ICPp como estrategia de reperfusión.

Es de destacar el número de pacientes que luego de fibrinólisis exitosa fueron referidos a intervención coronaria percutánea sistemática precoz como parte de una estrategia farmacoinvasiva y que en este grupo el éxito angiográfico fue mayor que en el grupo de pacientes que fueron sometidos a ICPp. El porcentaje de pacientes con esta estrategia fue mayor en el presente estudio comparado con el 12,9\% en PERSTEMI I, lo que finalmente hace que la estrategia de intervención coronaria percutánea sistemática precoz se consolide como una estrategia efectiva para la realidad de nuestro país.

Como reportan múltiples registros internacionales, confirmamos que la reducción del tiempo de isquemia total y del tiempo al primer contacto médico se asocian con reperfusión coronaria exitosa de forma estadísticamente significativa ${ }^{(28)}$. La presentación tardía y la ausencia de redes integradas para el manejo del IMCEST son problemas asociados con tiempos prolongados en nuestro medio, y en países en vías de desarrollo en general, que impiden mejorar los resultados clínicos ${ }^{(29)}$.

La mortalidad intrahospitalaria fue menor a la reportada en PERSTEMI I (8,6\% vs 10,1\%) la cual es comparable a la observada por otros registros como ARGEN-IAM-ST (8,7\%), Río Grande Brasil (8,9\%) y RENASICA III (8,7\%) aunque mayor a la reportada por PHASE-MX con 6,2\% para ICP y $4,8 \%$ para la estrategia percutánea sistemática precoz ${ }^{(15,17,18,19)}$. La mortalidad de causa cardíaca sigue siendo la más frecuente.

Más de la mitad de la población de estudio no fue reperfundida o la reperfusión no fue exitosa, lo que en el análisis de regresión se tradujo en mayores eventos adversos incluidos muerte en la evolución. Por lo anterior, es necesario reducir los tiempos de atención del IMCEST, lo que incluye educación a la población general, capacitación constante al primer nivel de atención, tener un solo sistema integrado de atención del IMCEST con centros derivadores y centros de mayor complejidad para intervencionismo, todos entrelazados por un sistema de transporte adecuado; solo así, se podrá lograr mayores tasas de reperfusión exitosa y menos eventos adversos. 
Como limitaciones del estudio podemos señalar que, si bien el registro PERSTEMI II se propuso tener alcance nacional, los datos obtenidos corresponden principalmente a centros hospitalarios públicos ubicados en la ciudad de Lima y el Callao, por lo que sus conclusiones no necesariamente reflejan la situación de los pacientes atendidos por IMCEST a nivel nacional. Así mismo, hay que considerar la influencia de la pandemia por COVID-19 sobre la atención y resultados clínicos de los pacientes con IMCEST en este registro.

\section{Conclusiones}

El IMCEST en el Perú se presenta con mayor frecuencia en varones en la séptima década de vida siendo los factores de riesgo más prevalentes la hipertensión arterial y la dislipidemia. El tratamiento de reperfusión más frecuente fue la fibrinólisis. El motivo de la falta de administración de terapia de reperfusión sigue siendo la llegada tardía de los pacientes a los servicios de salud con capacidad de administrar reperfusión. El éxito de la reperfusión se asoció con menor mortalidad cardiovascular, insuficiencia cardiaca, angina posinfarto y arresto cardiaco. Es necesaria la generación de un programa integrado de manejo del IMCEST a nivel nacional para mejorar los resultados clínicos.
Contribución de los autores: MCHD: concepción del artículo, recolección, análisis, redacción. RRO, DMN, PCS: recolección, análisis, redacción AMC,GYG,ARR,RIB,PRC,NLN,MLR,MG: recolección, redacción. AHV: análisis, redacción.

Agradecimiento: Dr. Carlos Pereda por la imagen central.

Investigadores PERSTEMI II: Roger Isla, Luis López (Hospital Alberto Sabogal - Callao), Paol Rojas, Germán Yabar (Hospital Guillermo Almenara -Lima), Aida Rotta (Hospital Cayetano Heredia - Lima), Javier Chumbe (Hospital Arzobispo Loayza - Lima), Rubén Azañero (Hospital 2 de mayo, Lima), Mauricio García (Hospital Hipólito Unanue, Lima), Carlos Barrientos (Hospital MINSA-Huancayo), Jorge Martos (Hospital MINSA Cajamarca), Alexander Montesinos, Fernando Gamio (Hospital Adolfo Guevara-Cusco), Nassip Llerena (Hospital Carlos Seguín-Arequipa), Piero Custodio (Hospital Almanzor Aguinaga-Chiclayo), Julio Uribe (Hospital Essalud- Iquitos), Walter Saavedra (Hospital Essalud-Tumbes), Fernando Allende (Hospital Essalud- Puno), Pamela Mejía (Hospital EssaludTacna), René Rodriguez, David Miranda y Manuel Chacón (Instituto Nacional Cardiovascular, INCOR).

\section{Referencias bibliográficas}

1. World Health Organization. World Health Statistics 2011. WHO's annual compilation of data from its 193 Member States, including a summary of progress towards the health-related Millennium Development Goals and Targets. Geneva: WHO; 2011.

2. Lloyd-Jones D, Adams R, Carnethon M, De Simone G, Ferguson TB, Flegal $\mathrm{K}$, et al. Heart disease and stroke statistics - 2009 update: a report from the American Heart Association Statistics Committee and Stroke Statistics Subcommittee. Circulation. 2009;119(3):e21181. doi: 10.1161/CIRCULATIONAHA.108.191261.

3. O'Gara PT, Kushner FG, Ascheim DD, Casey Jr DE, Chung MK, De Lemos JA, et al. 2013 ACCF/AHA guideline for the management of ST-elevation myocardial infarction: a report of the American College of Cardiology Foundation/American Heart Association Task Force on Practice Guidelines. J Am Coll Cardiol. 2013;61(4):e78-e140. doi: 10.1016/j.jacc.2012.11.019.

4. Gharacholou SM, Alexander KP, Chen AY, Wang TY, Melloni C, Gibler $W B$, et al. Implications and reasons for the lack of use of reperfusion therapy in patients with ST-segment elevation myocardial infarction: findings from the CRUSADE initiative. Am Heart J. 2010;159(5):757-63. doi: 10.1016/j.ahj.2010.02.009.

5. Fuster V, Badimon L, Badimon JJ, Chesebro JH. The pathogenesis of coronary artery disease and the acute coronary syndromes (part I). N Engl J Med. 1992;326(5):310-8. doi: 10.1056/NEJM199201303260506.

6. Ibañez B, James S, Agewall S, Antunes MJ, Bucciarelli-Ducci C, Bueno $\mathrm{H}$, et al. 2017 ESC Guidelines for the management of acute myocardial infarction in patients presenting with ST-segment elevation:The Task Force for the management of acute myocardial infarction in patients presenting with ST-segment elevation of the European Society of Cardiology (ESC). Eur Heart J. 2018;39(2):119-177. doi: 10.1093/ eurheartj/ehx393.
7. Gagliardi J, CHARASK A, Perna E, D'Imperio H, Bono J, Castillo Y, et al. Encuesta nacional de infarto agudo de miocardio con elevación del segmento ST en la República Argentina (ARGEN-IAM-ST) Rev Argent Cardiol. 2016;84(6):548-557.

8. Garcia A, Jerjes-Sanchez C, Martinez P, Azpiri-López JR, Caballero AA, Martínez C, et al. Renasica II. Un registro mexicano de los síndromes coronarios agudos. Arch Cardiol Mex. 2005;75(supl 2):S6-S19.

9. Chacón M, Vega A, Aráoz O, Ríos P, Baltodano R, Villanueva F, et al. Características epidemiológicas del infarto de miocardio con elevación del segmento ST en Perú: resultados del PEruvian Registry of ST-segment Elevation Myocardial Infarction (PERSTEMI). Arch Cardiol Mex. 2018;88(5):403-412.

10. Higa CC, D'Imperio H, Blanco P, Charask A, Arazi HC, Novo F, et al. Comparación de dos registros argentinos de infarto de miocardio: SCAR 2011 y ARGEN-IAM ST 2015. Rev Argent Cardiol. 2019;87(1):1925. doi: 10.7775/rac.es.v87.i1.14515.

11. Brindis RG, Bates ER, Henry TD. Value of Registries in ST-SegmentElevation Myocardial Infarction Care in Both the Pre-Coronavirus Disease 2019 and the Coronavirus Disease 2019 Eras. J Am Heart Assoc. 2021;10:e019958. doi: 10.1161/JAHA.120.019958.

12. Custodio-Sánchez P, Miranda D, Murillo L. Impacto de la Pandemia por COVID-19 sobre la Atención del Infarto de Miocardio ST Elevado en el Perú. Arch Peru Cardiol Cir Cardiovasc. 2020;1(1):87-94. doi: 10.47487/apcyccv.v1i2.22.

13. Rodriguez-Leor O, Cid-Álvarez B, Ojeda S, Martín-Moreiras J, Rumoroso JR, López-Palop R, et al. Impacto de la pandemia de COVID-19 sobre la actividad asistencial en cardiología intervencionista en España. REC Interv Cardiol. 2020;2(2):82-89. doi: 10.24875/RECIC.M20000120. 
14. Garcia S, Albaghdadi M, Meraj PM, Schmidt C, Garberich R, Jaffer FA, et al. Reduction in ST-Seg-ment Elevation Cardiac Catheterization Laboratory Activations in the United States during COVID-19 Pandemic. J Am Coll Cardiol. 2020;75(22):2871-72. doi: 10.1016/j. jacc.2020.04.011.

15. D'Imperio H, Gagliardi J, Charask A, Zoni R, Quiroga W, Castillo Y, et al. Infarto agudo de miocardio con elevación del segmento ST en la Argentina. Datos del registro continuo ARGEN-IAM-ST. Rev Argen Cardiol. 2020;88(4):297-307. doi: 10.7775/rac.es.v88.i4.18501.

16. Filgueiras NM, Feitosa GS, Fontoura DJ, Coelho F, Oliveira P, Paiva I, et al. Implementation of a Regional Network for ST-Segment-Elevation Myocardial Infarction (STEMI) Care and 30-Day Mortality in a Lowto Middle-Income City in Brazil: Findings From Salvador's STEMI Registry (RESISST). J Am Heart Assoc. 2018;7:e008624. doi: 10.1161/ JAHA.118.008624.

17. Alves L, Polanczyk CA. Hospitalization for Acute Myocardial Infarction: A Population-Based Registry. Arq Bras Cardiol. 2020;115(5):916-924. doi: 10.36660/abc.20190573.

18. Martinez-Sanchez C, Borrayo G, Carrillo J, Juarez U, Quintanilla J, Jerjes-Sanchez $C$, et al. Clinical management and hospital outcomes of acute coronary syndrome patients in Mexico: The Third National Registry of Acute Coronary Syndromes (RENASICA III). Arch Cardiol Mex. 2016;86(3):221-232. doi: 10.1016/j.acmx.2016.04.007.

19. Araiza-Garaygordobil D, Gopar-Nieto R, Cabello-López A. Pharmacoinvasive Strategy vs Primary Percutaneous Coronary Intervention in Patients With ST-Elevation Myocardial Infarction: Results From a Study in Mexico City. CJC Open. 2021;3(4):409-418. doi: 10.1016/j.cjco.2020.11.012

20. Farré N, Fort A, Tizón-Marcos H, Recasens L, Vaquerizo B, Serrat R, et al. Epidemiology of heart failure in myocardial infarction treated with primary angioplasty: Analysis of the Codi IAM registry. REC Cardio Clin. 2019;54(1):41-49. doi: 10.1016/j.rccl.2019.01.014

21. Guzmán-Rodríguez R, Polo-Lecca G, Aráoz-Tarco O, Alayo-Lizama C, Chacón-Díaz M. Características Actuales y Factores de Riesgo de Mortalidad en Choque Cardiogénico por Infarto de Miocardio en un Hospital Latinoamericano. Arch Peru Cardiol Cir Cardiovasc. 2021;1(4). doi: 10.47487/apcyccv.v1i4.89.
22. Zeymer W, Bueno H, Granger CB, Hochman J, Huber K, Lettino M, et al. Acute Cardiovascular Care Association position statement for the diagnosis and treatment of patients with acute myocardial infarction complicated by cardiogenic shock: A document of the Acute Cardiovascular Care Association of the European Society of Cardiology. Eur Heart J Acute Cardiovasc Care. 2020;9(2):183-197. doi: $10.1177 / 2048872619894254$.

23. Belle L, Cayla G, Cottin Y, Coste P, Khalife K, Labeque JN, et al. French Registry on Acute ST-elevation and non-ST-elevation Myocardial Infarction 2015 (FAST-MI 2015). Design and baseline data. Arch Cardiovasc Dis. 2017;110(6-7):366-378. doi: 10.1016/j. acvd.2017.05.001.

24. American College of Emergency Physicians. Public Poll: Emergency Care Concerns amidst COVID-19. US: ACEP; 2020. Disponible en: https://www.emergencyphysicians.org/article/covid19/public-pollemergency-care-concerns-amidst-covid-19

25. Tam C, Cheung K, Lam S, Wong A, Yung A, Sze M, et al. Impact of Coronavirus Disease 2019 (COVID-19) Outbreak on ST-SegmentElevation Myocar-dial Infarction Care in Hong Kong, China. Circ Cardiovasc Qual Outcomes. 2020;13(4):1-3. doi: 10.1161/ CIRCOUTCOMES.120.006631.

26. Jing ZC, Zhu HD, Yan XW, Chai WZ, Zhang S. Recommendations from the Peking Union Medical College Hospital for the management of acute myocardial infarction during the COVID-19 outbreak. Eur Heart J. 2020;41(19):1791-1794. doi: 10.1093/eurheartj/ehaa258.

27. De Luca G, Verdoia M, Cercek M, Jensen LO, Vavlukis M, Calmac L, et al. Impact of COVID-19 Pandemic on Mechanical Reperfusion for Patients With STEMI. J Am Coll Cardiol. 2020;76(20):2321-2330. doi: 10.1016/j.jacc.2020.09.546

28. Ibañez B, James S, Agewall S, Antunes MJ, Bucciarelli-Ducci C, Bueno $H$, et al. Guía ESC 2017 sobre el tratamiento del infarto agudo de miocardio en pacientes con elevación del segmento ST. Rev Esp Cardiol. 2017;70(12):1039-1045. doi: 10.1016/j.recesp.2017.10.048.

29. Mehta S, Granger C, Lee Grines C, Jacobs A, Henry TD, Rokos I, et al. Confronting system barriers for ST- elevation $\mathrm{MI}$ in low- and middle-income countries with a focus on India. Indian Heart J. 2018;70(1):185-190. doi: 10.1016/j.ihj.2017.06.020. 